Check for updates

Cite this: RSC Adv., 2019, 9, 20019
Received 21st May 2019

Accepted 13th June 2019

DOI: 10.1039/c9ra03813b

rsc.li/rsc-advances

\section{Dicarbonyl-tuned microstructures of hierarchical porous carbons derived from coal-tar pitch for supercapacitor electrodes}

\begin{abstract}
Haiyang Wang, ${ }^{\mathrm{ab}}$ Hongzhe Zhu, (D) ${ }^{\mathrm{b}}$ Shoukai Wang, ${ }^{\text {*b }}$ Debang Qia and Kaihua Shen ${ }^{\star a}$
A simple and effective template-free method to prepare hierarchical porous carbons (HPCs) has been developed by using low-cost coal-tar pitch as a starting material, anhydrous aluminum chloride as the Friedel-Crafts catalyst, and oxalyl chloride as the cross-linking agent. By a simple controllable FriedelCrafts reaction, diketone-functionalized coal-tar pitch as the hierarchical porous coal-tar pitch precursor was obtained via a one-step carbonization to provide a well-developed micro-mesoporous network. Nitrogen adsorption and desorption measurements showed that the surface area, pore volume, pore size and pore size distributions of the resulting carbon materials was dependent on the usage of the crosslinking agent. The as-fabricated HPCs have a large Brunauer-Emmett-Teller specific surface area of $1394.6 \mathrm{~m}^{2} \mathrm{~g}^{-1}$ and exhibit an excellent electrochemical performance with the highest specific capacitance of $317 \mathrm{~F} \mathrm{~g}^{-1}$ at a current density of $1 \mathrm{~A} \mathrm{~g}^{-1}$ in a three-electrode system. A symmetric supercapacitor was fabricated from HPC-DK-1.0 in a two-electrode system, which exhibits a high specific capacitance of $276 \mathrm{~F}$ $\mathrm{g}^{-1}$ at a current density of $0.25 \mathrm{~A} \mathrm{~g}^{-1}$, a high rate capability and an excellent cycling stability with a capacitance retention of $92.9 \%$ after 10000 cycles. The one-step carbonization method that produced HPCs for electrical double-layer capacitors represents a new approach for high-performance energy storage.
\end{abstract}

\section{Introduction}

In recent years, the design and preparation of organic hierarchical porous carbons (HPCs) has attracted significant attention in academia and industry because of the HPCs unique nanoporous hierarchy, which has potential application in catalysis, gas separation, electromagnetic interface shielding, supercapacitors and fuel cells etc. ${ }^{1-5}$ To our knowledge, HPCs have been prepared by hard-soft-templating approaches or templating/corrosive-chemical-activation combination methods. ${ }^{6-9}$ These strategies have achieved great success in the preparation of various HPCs with precise pore structures. However, they have some limitations. For example, the procedure is complicated and tedious because of the required fabrication of templates with a special nanostructure or molecular structure, the removal of hard-templates or post-activation treatment, and many expensive templates are required. ${ }^{10-12}$ These limitations, result in an uncompetitive price-toperformance ratio for the HPCs compared with other materials for any given application, which limits their commercial viability. An exploration of new template-free preparation methods is urgently required in the study of HPCs.

${ }^{a}$ The State Key Laboratory of Fine Chemicals, Dalian University of Technology, Dalian, 116012, PR China. E-mail: shen_kh@dlut.edu.cn; Tel: +86-411-84986102

${ }^{b}$ Sinosteel Anshan Research Institute of Thermo-Energy Company Limited, Anshan, 114044, PR China. E-mail: wsk5840863@126.com
Coal-tar pitch (CP) is the main by product of the coking process in the coal chemical industry, and is often used to prepare carbon materials because of its relatively low price, sufficient quantity and higher carbon yield. For example, CP can be used to produce needle coke, carbon fibers, mesocarbon microbeads and carbon foam. ${ }^{13-16}$ In recent years, there has been growing interest in the application of porous carbons in the fields of gas storage and EDLC. ${ }^{17,18}$ However, preparation of pitch-based HPCs often requires templates or supports, such as mesoporous silica, metal oxides or silicon wafer that caps a metallic layer. ${ }^{19-22}$ The procedure is too complicated and tedious to apply in practice, therefore, it is imperative that new methods be developed to prepare pitch-based HPC carbons. One of the most widely used strategies to prepare HPCs is by the Friedel-Crafts reaction, in which pitch is polymerized under mild conditions and uses cheap and sustainable building blocks to produce highly porous hyper-cross-linked materials. ${ }^{23,24}$ Such polymers contain various molecules from oligomers to 3D cross-linking supramolecules with significant differences in molecular size, structure, and pyrolysis behavior. Some light molecules or thermolabile groups in the pitch are removed during the controlled pyrolysis, which could generate abundant mesopores. The devolatilization of the volatile component is accompanied by bubble formation, which occurs first in the vicinity of the primary bubble nuclei. The bubbles coalesce and grow under appropriate conditions, which leads to formation of mesopore voids or macropores in carbonized 
products. ${ }^{25}$ Thus, the pitch polymers may be promising candidates for constructing HPCs with explicit mesopore control for high-performance supercapacitors.

We report herein the template-free fabrication of a novel type of HPC by constructing diketone (-COCO-) cross-linking bridges between polycyclic aromatic hydrocarbons (PAH) in CP to yield diketone-functionalized CP (DKCP). The polar carbonyl group has a high reaction activity and favors the modification of CP by providing it with hydrophilic properties that enhance its wettability for polar solvents, and the oxygen functional groups can be used as anchoring sites for metal particles and large molecules. ${ }^{26,27}$ Such novel bridges can provide a high crosslinking density and oxygen atoms to the hierarchical porous modified CP materials, to achieve the carbonizability of a crosslinking modified CP framework and the inheritability of a hierarchical nanoporous structure. These properties should make DKCP a promising candidate for HPC production with the characteristics of preparation simplicity and easy scalability. The overall synthetic procedure is illustrated in Fig. 1.

\section{Experimental}

\subsection{Materials}

The raw CP was obtained from the Anshan Iron and Steel Group Co. Ltd (Anshan, China). A refined coal tar pitch (RFCP) with a softening point of $33{ }^{\circ} \mathrm{C}$ was obtained by a mixed solventextraction method and its compositions. The main properties of the RFCP and DKCP are shown in Table 1. Anhydrous aluminum chloride $\left(\mathrm{AlCl}_{3}\right)$ was from Tianjin Guangfu Fine Chemical Reagent Co. Ltd. (Tianjin, China). Oxalyl chloride (OC), hydrochloric acid ( $\mathrm{HCl}$ ) and dichloroethane (DCE) were from Sinopharm Chemical Reagent Co., Ltd. (Shanghai, China). All chemical reagents were of analytical grade.

\subsection{Procedures}

2.2.1 Polymerization of RFCP. In this one-step crosslinking approach, oxalyl chloride (OC) was used as an external cross-linker to react with RFCP. RFCP (50 g) was dissolved in $500 \mathrm{~mL}$ of 1,2-dichloroethane under argon, before a certain amount of $\mathrm{OC}$ and $\mathrm{AlCl}_{3}$ was added to the solution. The resultant mixture was stirred for $6 \mathrm{~h}$ at $40{ }^{\circ} \mathrm{C}$ to undergo the $\mathrm{AlCl}_{3}$ catalyzed Friedel-Crafts reaction of the RFCP and OC. The reaction was terminated by adding an ethanol-water solution. The product was filtered, washed with an ethanol-water solution that contained hydrochloric acid, and dried under a reduced pressure at $80{ }^{\circ} \mathrm{C}$ for $12 \mathrm{~h}$. The obtained DKCPs with different OC/RFCP mass ratios are referred to as DKCP- $x$, where $x$ represents the mass ratio of OC vs. RFCP. All resultant samples were dark-brown powders.

2.2.2 Carbonization. Sample carbonization was carried out in a tube furnace under atmospheric pressure according to the following procedures. Approximately $10 \mathrm{~g}$ of sample was carbonized at $800{ }^{\circ} \mathrm{C}$ for $2 \mathrm{~h}$ with a heating rate of $2{ }^{\circ} \mathrm{C} \mathrm{min}^{-1}$ to yield HPCs. The obtained HPC-DKs with different OC/RFCP mass ratios are referred to as HPC-DK- $x$, where $x$ represent the mass ratio of OC $v s$. RFCP. A $\mathrm{N}_{2}$ stream was introduced into the tube furnace throughout the carbonization.

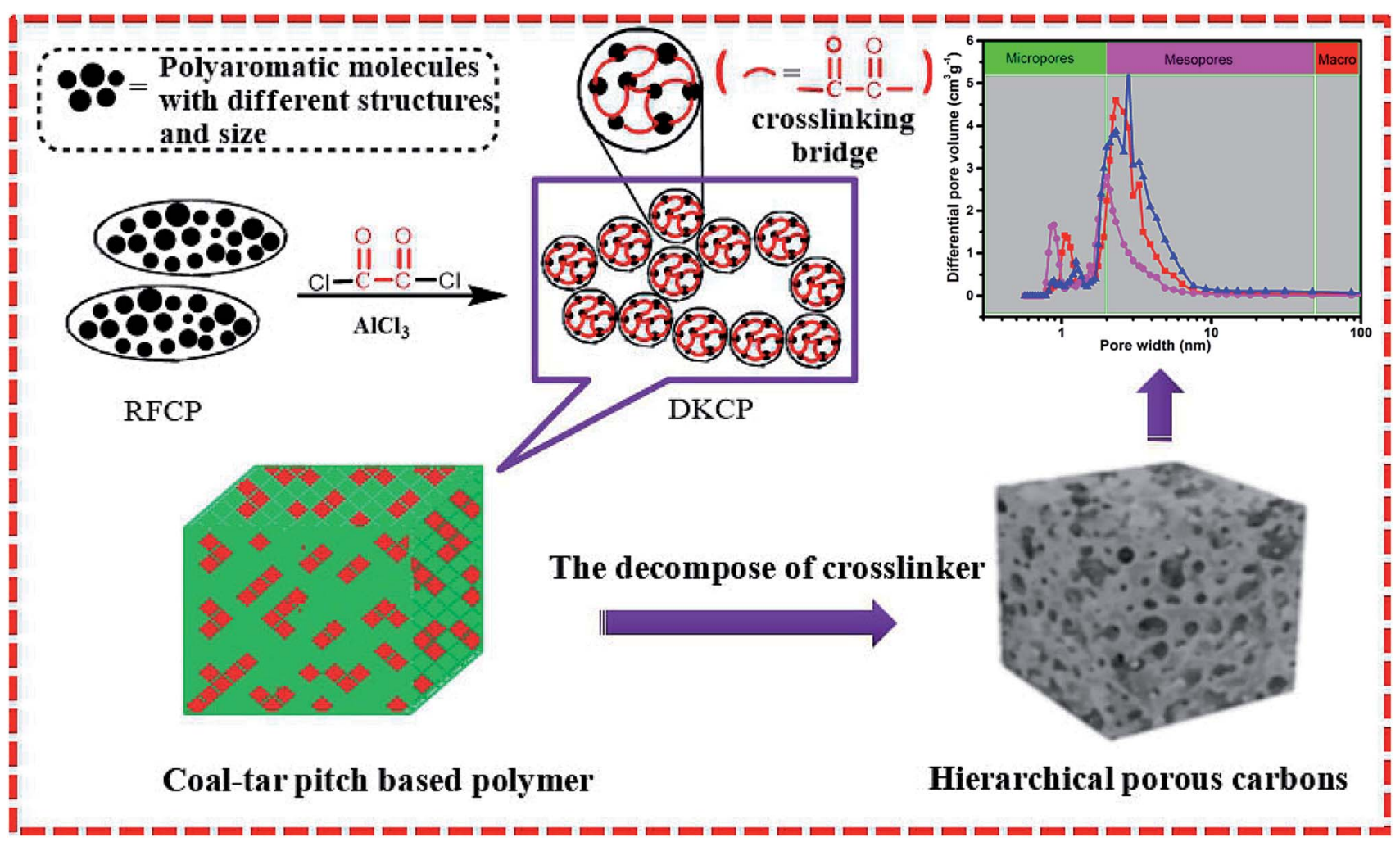

Fig. 1 Scheme of preparation mechanism of HPC materials in this study. 
Table 1 Bulk and surface elemental compositions of RFCP and DKCPs

\begin{tabular}{|c|c|c|c|c|c|c|c|c|}
\hline \multirow[b]{2}{*}{ Sample } & \multicolumn{6}{|c|}{ Element analysis (wt\%) } & \multicolumn{2}{|l|}{ XPS } \\
\hline & C & $\mathrm{H}$ & $\mathrm{O}$ & $\mathrm{N}$ & $\mathrm{C} / \mathrm{H}$ & $\mathrm{C} / \mathrm{O}$ & $\mathrm{C} / \mathrm{at} \%$ & $\mathrm{O} / \mathrm{at}$ \\
\hline RFCP & 93.2 & 4.7 & 1.9 & 0.2 & 1.65 & 65.4 & 95.2 & 4.6 \\
\hline DK-CP-0.5 & 80.7 & 3.3 & 15.8 & 0.2 & 2.04 & 7.86 & 84.6 & 13.7 \\
\hline DK-CP-1.0 & 73.6 & 2.6 & 23.6 & 0.2 & 2.36 & 4.16 & 83.2 & 15.7 \\
\hline DK-CP-1.5 & 70.2 & 2.1 & 27.5 & 0.2 & 2.79 & 3.40 & 82.8 & 16.1 \\
\hline
\end{tabular}

\subsection{Measurements and analyses}

Fourier transform-infrared (FTIR) spectra were collected on a Thermo Nicolet-360 spectrometer (USA). The elemental content of carbon, hydrogen, and chlorine were analyzed with a Vario Macro EL analyzer (Germany). Thermogravimetric analysis (TGA) was performed to determine the pyrolysis samples by using an HCT-1 instrument (China). Samples morphologies were observed by JSM-6700F scanning electron microscope (FESEM, Japan) and by using a Tecnai-G20 transmission electron microscopy (TEM, USA). Surface chemical composition of samples was studied by X-ray photoelectron spectroscopy (XPS, Thermo ESCALAB250, USA). The surface area and porosity of the samples were estimated from the isotherms of nitrogen adsorption-desorption at $77 \mathrm{~K}$ by ASAP2020. The specific surface area was calculated with the Brunauer-Emmett-Teller (BET) equation. The pore size distribution of the samples was calculated based on the density functional theory (DFT) method.

\subsection{Electrochemical measurements}

The carbon electrode was fabricated by mixing HPCs and polytetrafluoroethylene (PTFE) with a mass ratio of $9: 1$. Then, the mixture was rolled into a thin film and cut into round films (12 $\mathrm{mm}$ in diameter). Each round film with a $2.5 \mathrm{mg} \mathrm{cm}^{-2}$ mass loading was dried in vacuum oven at $120{ }^{\circ} \mathrm{C}$ for $2 \mathrm{~h}$, and then pressed onto nickel foams to fabricate supercapacitors electrodes. The obtained electrodes were soaked in $6 \mathrm{M} \mathrm{KOH}$ electrolyte under vacuum for $120 \mathrm{~min}$. A button type-supercapacitor was assembled by two similar electrodes and separated by a polypropylene membrane. The cyclic voltammetry (CV) and electrochemical impedance spectroscopy (EIS) measurements were conducted by using a CHI760E electrochemical workstation (Chenhua, Shanghai, China). EIS was carried out over a frequency range of $100 \mathrm{kHz}$ to $0.01 \mathrm{~Hz}$ with an amplitude of $5 \mathrm{mV}$. The galvanostatic charge-discharge measurements and cycle life tests were conducted on a supercapacitance test system (SCTs, Arbin Instruments, USA). The specific capacitance of the working electrodes was calculated from the galvanostatic discharge process via the following equation.

$$
\begin{gathered}
C_{\mathrm{s}}=\frac{I \times \Delta t}{m \times \Delta V} \\
C_{\text {cell }}=\frac{4 \times I \times \Delta t}{m \times \Delta V}
\end{gathered}
$$

where $C_{\mathrm{s}}\left(\mathrm{F} \mathrm{g}^{-1}\right)$ is the specific capacitances of the three electrodes system, $C_{\text {cell }}\left(\mathrm{F} \mathrm{g}^{-1}\right)$ is the specific capacitances of the symmetric supercapacitor system, $I$ is the discharge current (A), $\Delta t$ is the discharge time (s), $\Delta V$ is the voltage change $(V)$ that excludes the voltage drop during the discharge process, and $m$ is the mass of the active material (g).

The energy and power density of the symmetric supercapacitor systems were calculated by using the eqn (3) and (4):

$$
\begin{gathered}
E_{\text {cell }}=\frac{C_{\text {cell }} \times \Delta V^{2} \times 1000}{2 \times 4 \times 3600} \\
P_{\text {cell }}=\frac{E_{\text {cell }} \times 3600}{\Delta t}
\end{gathered}
$$

where $E_{\text {cell }}\left(\mathrm{W} \mathrm{h} \mathrm{kg}{ }^{-1}\right)$ is the specific energy density, $P_{\text {cell }}(\mathrm{W}$ $\left.\mathrm{kg}^{-1}\right)$ is the specific power density, $C_{\text {cell }}\left(\mathrm{F} \mathrm{g}^{-1}\right)$ is the total specific capacitance of the two-electrode cell, $\Delta V$ is the voltage change that excludes the IR drop during the discharge process, and $\Delta t$ is the discharge time.

\section{Results and discussion}

\subsection{Characteristics of RFCP and DKCPs}

3.1.1 Elemental analysis and XPS analysis. The elemental analysis of the RFCP and the DKCPs is provided in Table 1. The DKCPs compared with the RFCP have a high oxygen content with an increase in OC content. The decrease of the $\mathrm{C} / \mathrm{O}$ ratio indicates that the diketone-structure was successfully introduced into the RFCP (Table 1). The introduction of diketone functional groups is key to achieving a highly disordered carbon structure. The introduction of oxygen induces cross-linking of the RFCP structure, which prevents the melting and orderly rearrangement of the RFCP during the high-temperature carbonization process, and inhibits the graphitization process. The evolution of $\mathrm{CO}$ and $\mathrm{CO}_{2}$ during the high temperature process changes the microstructure of the carbon materials and plays a dual regulation role.

The surface chemistry of the DKCPs was studied by X-ray photoelectron spectrometric (XPS) measurement (Fig. 2a). The XPS survey spectra show two peaks at binding energies of $284.1 \mathrm{eV}$ and $531.5 \mathrm{eV}$, which correspond to $\mathrm{C} 1 \mathrm{~s}$ and $\mathrm{O} 1 \mathrm{~s}$, respectively, and suggest that all the samples contain a considerable number of oxygen-containing groups on their surfaces. During the carbonization process, the oxygen-containing groups in the pitch are unstable and can decompose to $\mathrm{CO}_{2}$ and $\mathrm{CO}$ during heat treatment and self-activation of the HPC, ${ }^{28,29}$ which can assist in creating additional pores.

Information on the chemical state of the elements anchored to the DKCPs surface was obtained from XPS. In the C1s spectrum of the DKCP-1.0 (Fig. 2b), peaks exist for different functional groups, namely $\mathrm{C}=\mathrm{C}, \mathrm{C}-\mathrm{C}$, and $\mathrm{C}-\mathrm{H}$ bonds $(284.3 \mathrm{eV})$, $\mathrm{C}=\mathrm{O}$ bonds $(286.5 \mathrm{eV})$, and $\mathrm{O}-\mathrm{C}=\mathrm{O}$ bonds $(288.4 \mathrm{eV}) .{ }^{30}$ The $\mathrm{O} 1 \mathrm{~s}$ spectrum of the DKCP-1.0 (Fig. 2c) can be fitted with two major component peaks. The peak at $531.8 \mathrm{eV}$ is attributed to $\mathrm{C}=\mathrm{O}$ bonds and the $533.1 \mathrm{eV}$ peak results from $\mathrm{O}-\mathrm{C}=\mathrm{O}^{31}$ These observations show that the DKCPs consists of aromatic carbon with carbonyl and carboxylic functional groups. 

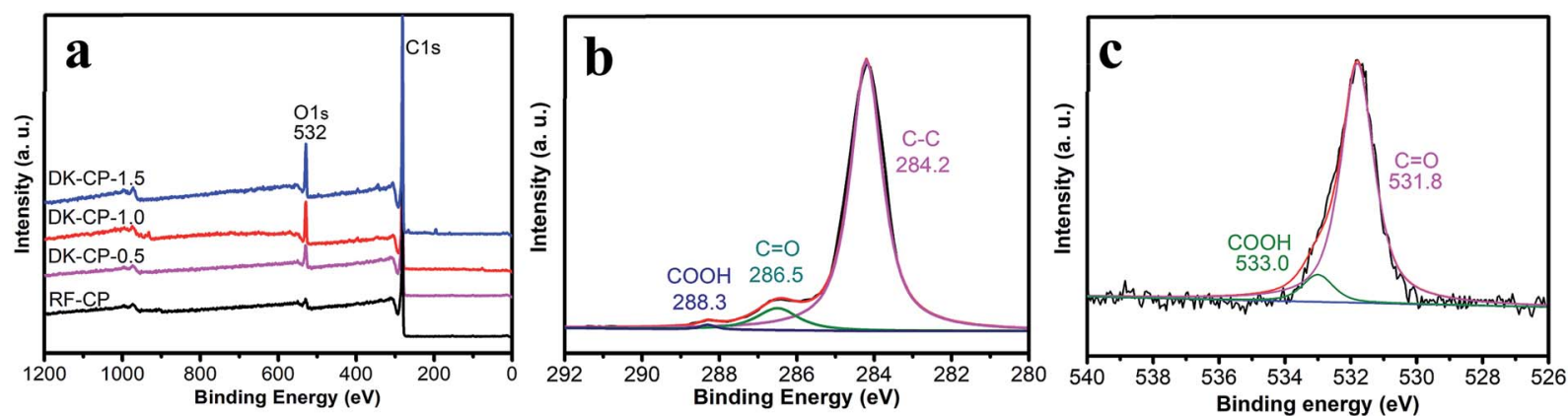

Fig. 2 XPS spectra for RFCP derivatives (a) C1s (b) O1s, and (c) XPS spectra of DKCP-1.0.

3.1.2 FT-IR analysis. As shown in Fig. 3, the absorption peaks at 3040 and $2910 \mathrm{~cm}^{-1}$ result from aromatic $\mathrm{C}-\mathrm{H}$ stretching vibration and aliphatic $\mathrm{C}-\mathrm{H}$ stretching vibration, respectively. ${ }^{32}$ The peak at $1600 \mathrm{~cm}^{-1}$ is attributed to aromatic $\mathrm{C}=\mathrm{C}$ stretching vibration and the peak at $1460 \mathrm{~cm}^{-1}$ is attributed to the $\mathrm{C}-\mathrm{H}$ bending vibration of methyl and methylene. ${ }^{33}$ The peaks at $1720 \mathrm{~cm}^{-1}$ is attributed to $\mathrm{C}=\mathrm{O}$ vibration. ${ }^{34}$ The peaks at $1170 \mathrm{~cm}^{-1}$ is attributed to $\mathrm{C}-\mathrm{C}$ stretching vibration. The absorption peaks of the aromatic ring skeletal vibrations move to lower wavenumbers after modification, which indicates the degree of conjugation of the aromatic rings. Hence, the degree of polymerization for the aromatic rings increases significantly. Therefore, RFCP can be bridged by OC.

3.1.3 Thermogravimetric analysis. Thermogravimetric analysis (TGA) was used with derivative thermogravimetry (DTG) to study the transitions of RFCP and DKCPs at different carbonization temperatures. Fig. 3b shows that both RFCP and DKCPs decompose in a single mass loss stage from 50 to $800^{\circ} \mathrm{C}$. The mass loss results mainly from the removal of gases and light compounds that are generated via thermal polymerization and the cracking of side chains of aromatic rings. ${ }^{35}$ The carbonization yields of RFCP, DKCP-0.5, DKCP-1.0 and DKCP1.5 are $16.3 \%, 41.5 \%, 55.9 \%$, and $65.9 \%$, respectively, which indicates that the carbonization yield of RFCP can be improved by cross-linking OC. This outcome can be rationalized as follows: OC can react with small molecules in RFCP to from large molecules, ${ }^{36}$ which decreases the removal of light compounds and increases the carbonization yield. The polar oxygen-containing functional groups from more thermalresistant materials and increase the carbonization yield of
RFCP. DTG curves (Fig. 3c) show that RFCP and DKCPs lose mass at varying rates, as related to the cross-linking degree by OC. The RFCP profile is characterized by a single peak that is centered at $251{ }^{\circ} \mathrm{C}$, which indicates that the mass loss rate at this temperature reaches a maximum. However, three peaks exist in the DTG profile of the DKCPs, which indicates that the main reactions/transformations that occur during the pyrolysis process are similar. The first peak centered at $224{ }^{\circ} \mathrm{C}$ (peak I) can be ascribed to the evaporation of absorbed water. Most oligomers in the RFCP fraction are easy to gasify and/or distill below $430{ }^{\circ} \mathrm{C},{ }^{37}$ which leads to the formation of a DTG peak that is centered at approximately $367^{\circ} \mathrm{C}$ (peak II). Above $450{ }^{\circ} \mathrm{C}$, the remaining oligomers and macromolecules cross-link to form larger molecules, even solidified coke. The condensation reactions are accompanied by a release of small molecules, such as $\mathrm{CO}_{2}, \mathrm{CO}, \mathrm{H}_{2} \mathrm{O}$, and $\mathrm{CH}_{4}$, that leads to the third DTG peak that is centered at approximately. $509{ }^{\circ} \mathrm{C}$ (peak III). ${ }^{38,39}$ When the temperature exceeds $620^{\circ} \mathrm{C}$, a carbon-structure rearrangement occurs in the solidified coke and no significant mass loss appears. $^{40}$ The configuration of small oligomers and large macromolecule networks in the diketone-functionalized pitch polymers leads to a stepwise pyrolysis and aggregation process, which could have a significant effect on the morphology and microstructure of the resultant carbons.

\subsection{Characterization of porous structure of HPCs}

3.2.1 Brunauer-Emmett-Teller (BET). Porous structures of HPC-RF and HPC-DKs are presented in Fig. 4a by measuring the $\mathrm{N}_{2}$ adsorption-desorption isotherms. All samples display
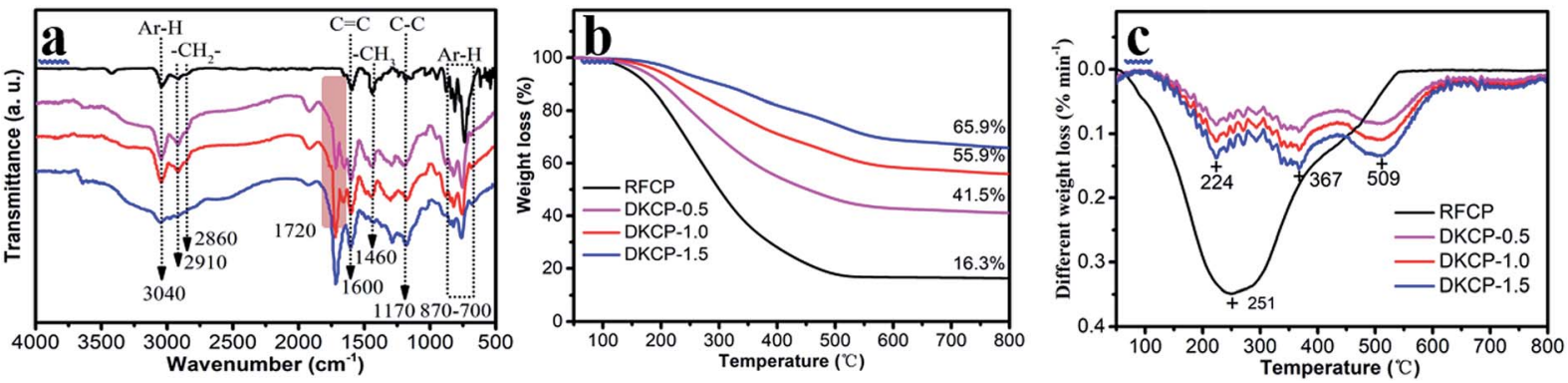

Fig. 3 IR spectrum of RFCP derivatives (a), TGA curves of RFCP derivatives (b), and DTG curves of CP derivatives (c). 

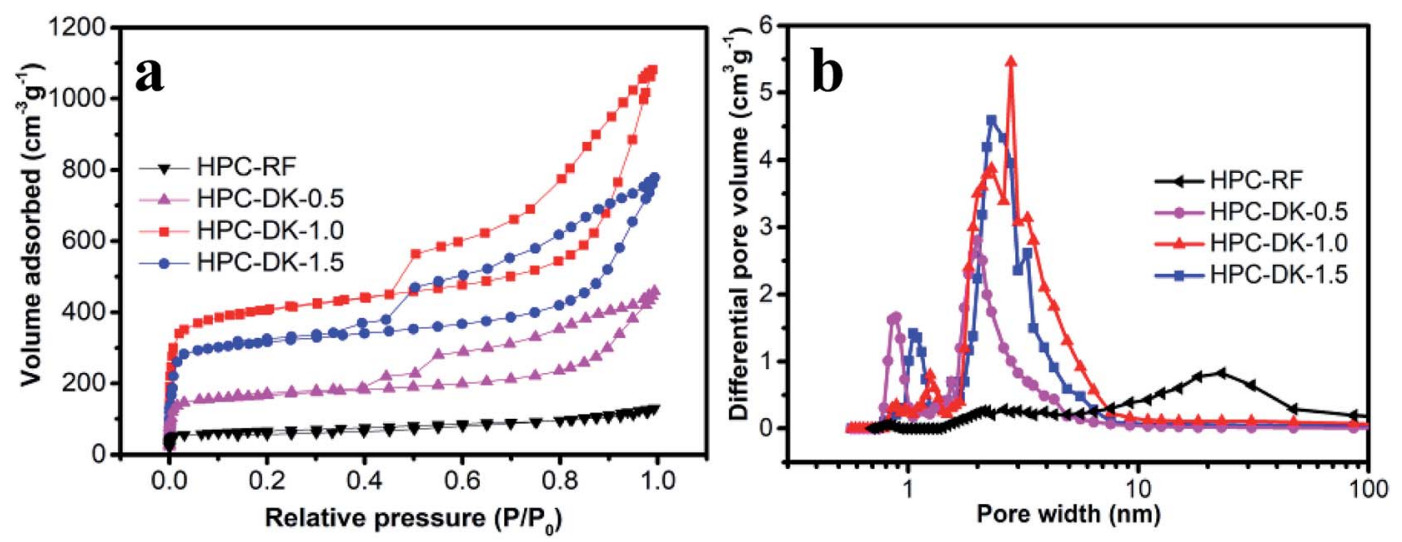

Fig. 4 (a) $\mathrm{N}_{2}$ absorption/desorption isotherms; (b) pore size distributions for the HPC samples.

mixture-type isotherms with hysteresis loops, which indicates a combination of microporous/mesoporous structure. ${ }^{41}$ The $\mathrm{N}_{2}$ adsorption isotherms of all HPC-DK samples show a steep $\mathrm{N}_{2}$ uptake at low relative pressure $\left(P / P_{0}<0.001\right)$, reflecting the existence of abundant micropores. The adsorption isotherms of HPC-DKs show an evident hysteresis loop in the medium pressure region $\left(P / P_{0}=0.4-0.9\right)$, indicating that a large number of mesopores exist in these HCPs. Therefore, the HPC-DK-0.5 shows a dominant pore size distribution of less than $2 \mathrm{~nm}$. With an increase of OC mass, a more porous structure emerges in the HPC-DK-1.5 and HPC-DK-1.0. The HPC-DK-1.0 exhibits a more significant hysteresis than HPC-DK-0.5, HPC-DK-1.5, and the HPC-RF in the relative pressure $\left(P / P_{0}\right)$ range of $0.4-$ 0.9 , which indicates a higher amount of mesopores in HPC-DK1.0 compared with other HPCs when using the same starting materials and for the same procedure. The porous structure was created by the crosslinking reactions and the heteroatoms in the carbonyl-functionalized pitch are chemically unstable, which provides more "active sites" for carbonization. Thus, the surface functional groups of the RFCP provide a meaningful contribution to the high BET surface area. The calculated structure parameters of a series of carbon materials, including the BET specific surface area (SSA), total and micro-mesopore volume are summarized in Table 2. For a constant sintering temperature, the SSA, pore size distribution, and pore volume of the HPCs are influenced significantly by the mass ratio of the cross-linking agent (OC). It is found that the specific surface areas and pore volume vary with the weight ratios of oxylyl chloride to refine coal-tar pitch (OC/RFCP). The highest BET surface area and pore volume $\left(1394.6 \mathrm{~m}^{2} \mathrm{~g}^{-1}, 1.54 \mathrm{~cm}^{3} \mathrm{~g}^{-1}\right)$ are obtained in HPC-DK-1.0. With the increase of OC/RFCP from 0.5 to 1.0 , the BET surface area increases from $410.9 \mathrm{~m}^{2} \mathrm{~g}^{-1}$ to
$1394.6 \mathrm{~m}^{2} \mathrm{~g}^{-1}$, and the pore volume from $0.64 \mathrm{~cm}^{3} \mathrm{~g}^{-1}$ to 1.54 $\mathrm{cm}^{3} \mathrm{~g}^{-1}$. However, a further increase of OC/RFCP leads to a decrease in the specific surface area and pore volume. Therefore, the larger BET surface area and pore volume at large OC/RFCP can be explained as the results of higher crosslinking degree. However, a further increase of OC/RFCP leads to a decrease in the specific surface area. The reason is that when excess OC is used, only one of the two acyl chloride groups in oxalyl chloride reacts with the aromatic rings. As a result, the crosslinking degree of RFCP decreases. The other acyl chloride group on oxalyl chloride is converted into a carboxyl group, as demonstrated by the FTIR spectra of the DKCPs. Fig. $4 \mathrm{~b}$ shows the pore-size distribution of the HPCs, which justifies the effectiveness of the micropore and mesopore introduction on the HPCs by a one-step carbonization process in the presence of a cross-linking agent (OC). The HPC-DK-1 shows a dominant pore size distribution of less than $2 \mathrm{~nm}$. With an increase in OC mass, a more porous structure emerges in the HPC-DK-1.5 and HPC-DK-1.0. The amount of $3 \mathrm{~nm}$ mesopores also increased, which provides a low resistant ionic pathway, and improves the accessibility of the micropores to electrolytes. The number of micropores less than $2 \mathrm{~nm}$ increased greatly for the HPC-DK-1.5 and HPC-DK-1.0 samples, as shown in Fig. 4b. The variety of pore sizes with a high pore volume provides highly efficient mass transport through the mesopores and a large SSA from the micro-to mesopores, which achieves an excellent performance for electrical double-layer capacitor applications.

3.2.2 SEM and TEM analysis. The HPC morphologies were studied by field-emission scanning-electron microscopy and high-resolution transmission-electron microscopy (HRTEM) (Fig. 5). The (fold)block structure for the RFCP after carbonization was clearly visible, and the mesopore and macropore

Table 2 Pore structure parameters of nanoporous carbon materials

\begin{tabular}{lcclll}
\hline Samples & $S_{\text {BET }}\left(\mathrm{m}^{2} \mathrm{~g}^{-1}\right)$ & $S_{\text {meso }}\left(\mathrm{m}^{2} \mathrm{~g}^{-1}\right)$ & $V_{\text {total }}\left(\mathrm{cm}^{3} \mathrm{~g}^{-1}\right)$ & $V_{\text {micro }}\left(\mathrm{cm}^{3} \mathrm{~g}^{-1}\right)$ & 0.003 \\
\hline HPC-RF & & & & 0.06 & $V_{\text {meso }}\left(\mathrm{cm}^{3} \mathrm{~g}^{-1}\right)$ \\
HPC-DK-0.5 & 127.6 & 24.9 & 0.64 & 0.26 \\
HPC-DK-1.0 & 410.9 & 138.5 & 1.54 & 0.41 & 0.38 \\
HPC-DK-1.5 & 1394.6 & 778.9 & 0.99 & 0.32
\end{tabular}



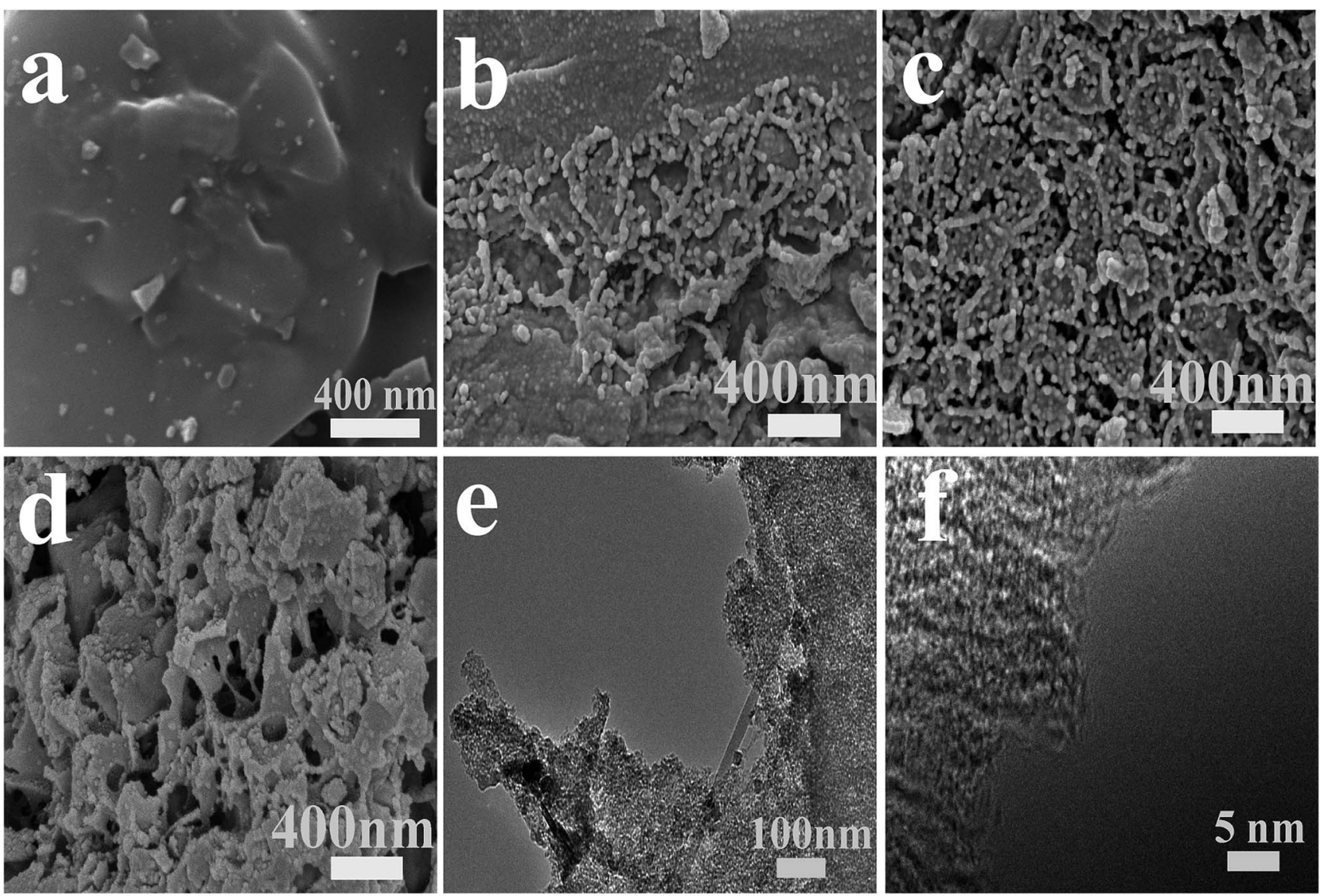

Fig. 5 SEM images of HPC-RF (a), HPC-DK-0.5 (b), HPC-DK-1.0 (c) and HPC-DK-1.5 (d), TEM images of HPC-DK-1.0 at $100 \mathrm{~nm}$ (e), and $5 \mathrm{~nm}$ (f).

structures appeared in their matrix as diketone groups that were introduced into the RFCP. The decomposition of carbonylfunctional RFCP was one of the factors that affected their porous nanostructure, and the carbonyl content is the main governing index. Holes were derived from the decomposition of the carbonyl group and the self-assembly foaming process, in which gases formed by pyrolysis/gasification of active precursor molecules lead to the formation of many holes, such as in common foaming processes for preparing carbon foams. ${ }^{42,43}$ This result is consistent with the composition characteristics of pitch cross-linked discussed above, in which the rich substituent carbonyl groups and the aromatic structures affect the fusibility of the DKCPs significantly, and thus determines the structural properties of the pyrolyzed carbons. The HPC-DK-0.5 contains more oligomers with a lower cross-linking degree, which possesses a lower systemic viscosity and a higher plasticity. DKCP-1.5, in contrast, has many macromolecules with serious cross-linking and so the number of holes on the HPCDK-1.5 surface is lower (Fig. 5d). The porous structure was verified by HRTEM. The HRTEM micrographs in Fig. 5e and $\mathrm{f}$ show that HPC-DK-1.0 is amorphous and has a highly disordered pore structure. Abundant micropores occur with the mesoporous channel walls, which indicates the formation of a continuous three dimensional pore network. This result is consistent with the DFT pore size distribution results.

\subsection{Electrochemical properties}

The electrochemical performances of the obtained carbon materials were investigated in a three-electrode system in $6.0 \mathrm{M}$ $\mathrm{KOH}$ solution. Cyclic voltammetry (CV) curves (Fig. 6a) of the carbonized material electrodes with a scanning rate of $50 \mathrm{mV}$ $\mathrm{s}^{-1}$ exhibited a typical rectangular $I-V$ curve without any redox peak with bumps -1.0 to $0 \mathrm{~V}$, which suggests that all carbonized materials exhibited a pure capacitive behavior. ${ }^{44,45}$ The HPC-DK1.0 presented the largest encircling area of the CV curve, which revealed its highest capacitance among the three HPC carbons. Galvanostatic charge-discharge (GCD) curves of HPCs obtained at $1 \mathrm{~A} \mathrm{~g}^{-1}$ (Fig. 6b) showed almost symmetrical triangles with a tiny deformation, which suggests a reversible electrochemical capacitive performance with a high charge/discharge efficiency. The capacitance of HPC-DK-1.0 calculated from the galvanostatic charge-discharge curve is $317 \mathrm{~F} \mathrm{~g}^{-1}$ at $1 \mathrm{~A} \mathrm{~g}^{-1}$ and is significantly higher than that of the carbon materials reported in previous studies. ${ }^{6,9,16,37}$ The large specific capacitance may be caused by its high accessible surface areas and rich reasonable distributed pores. More significantly, the IR-drops of the HPCs at the start of discharge are less than $7 \mathrm{mV}$, which suggests a very low equivalent series resistance, an excellent conductivity, and a high mass transfer and/or diffusion rate of ions within the electrode materials. Fig. $6 \mathrm{c}$ presents the rate performances of all the carbonized materials. The specific capacitances decrease 

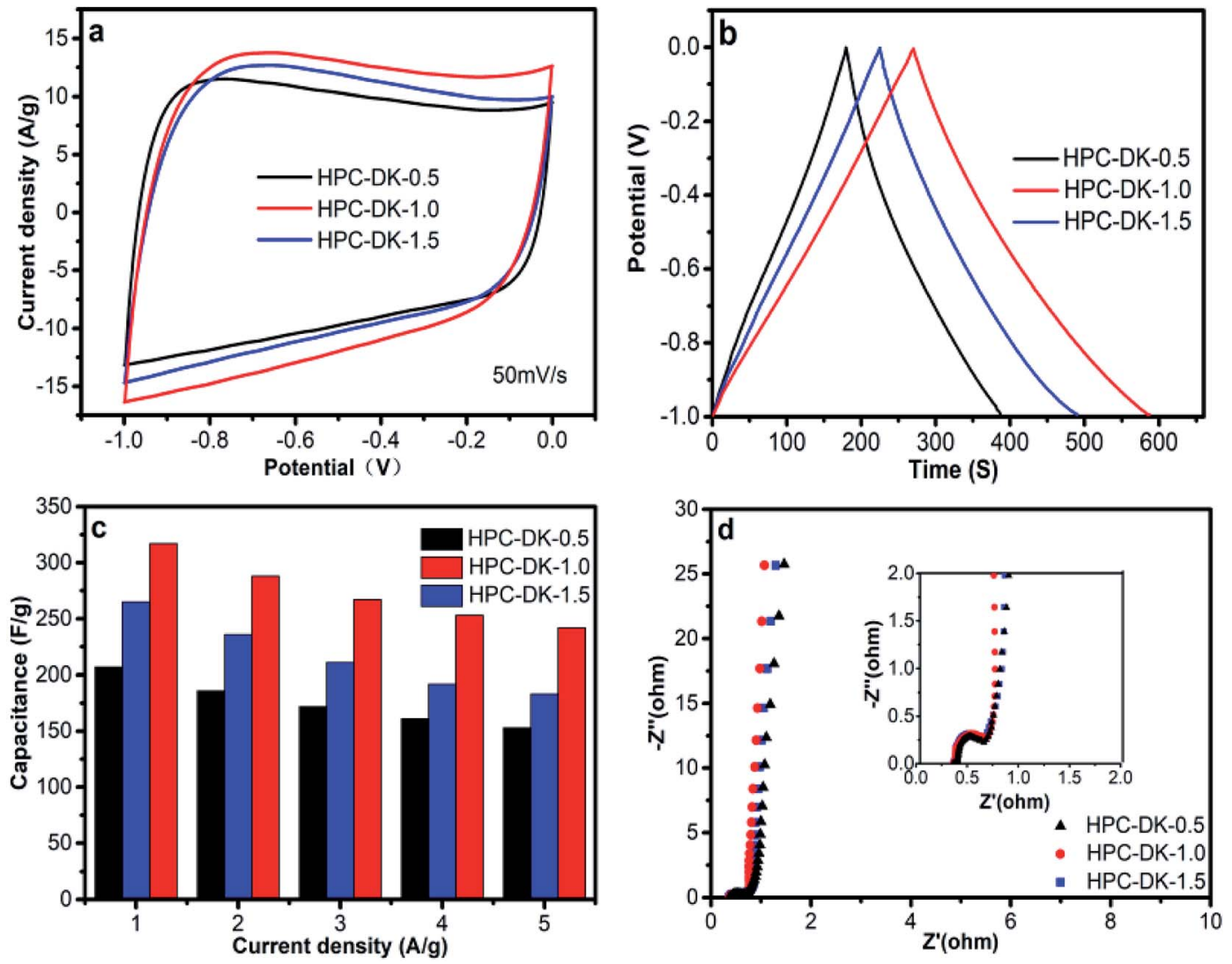

Fig. 6 Electrochemical performance of carbonized material based electrode measurement in a three-electrode system in $6.0 \mathrm{M} \mathrm{KOH}$ aqueous electrolyte. (a) CV curves at $50 \mathrm{mV} \mathrm{s}^{-1}$, (b) charge-discharge profiles at $1 \mathrm{~A} \mathrm{~g}^{-1}$, (c) capacitance at different current densities, and (d) Nyquist plots of porous carbon electrodes with inset showing plots in high frequency region.

with an increase in current density for all samples. The behavior is related closely to the pore-size dependent diffusion limitation of ions inside the electrode material at higher current densities. ${ }^{46}$ HPC-DK-1.0 exhibits the highest capacitance of $317 \mathrm{~F} \mathrm{~g}^{-1}$ at a current density of $1 \mathrm{~A} \mathrm{~g}^{-1}$ among the three carbonized materials, which is much higher than that of HPC-DK-0.5 (207 F $\left.\mathrm{g}^{-1}\right)$ and HPC-DK-1.5 $\left(265 \mathrm{~F} \mathrm{~g}^{-1}\right)$ at the same conditions. At a high current density of $10 \mathrm{~A} \mathrm{~g}^{-1}$, HPC-DK-1.0 exhibits a high specific capacitance of $242 \mathrm{~F} \mathrm{~g}^{-1}$, and retains $76.3 \%$ of the specific capacitance. The capacitance retentions in the same current range are $73.9 \%$ and $69.1 \%$ for HPC-DK-0.5 and HPCDK-1.5, respectively. The high specific capacitance and excellent rate performance of the HPC-DK-1.0 is ascribed to its superhigh BET surface area $\left(S_{\mathrm{BET}}=1394.6 \mathrm{~m}^{2} \mathrm{~g}^{-1}\right)$ and welldistributed hierarchical porous structures which provide a high accessible surface for electron accommodation and convenient electrolyte-ion transportation. ${ }^{47,48}$ Electrochemical impedance spectroscopy (Fig. 6d) was conducted to understand the capacitance mechanism. In the low frequency region, the line that is nearly parallel to the imaginary axis demonstrates an excellent supercapacitor capacitive behavior. ${ }^{49}$ In the medium frequency region, the inclined line with a $45^{\circ}$ slope corresponds to the diffusive resistance of electrolyte ions within the pores of electrode materials (Warburg resistance). In the high frequency region, the semicircle diameter indicates a change in transport resistance $\left(R_{\mathrm{ct}}\right)$ at the electrode/electrolyte interface. ${ }^{50}$ The intercept of the semicircle with a real axis $\left(z^{\prime}\right)$ is referred to as the internal resistance $\left(R_{\mathrm{s}}\right)$, which includes the intrinsic resistance of the electrode material, the contact resistance between the electrode material and the current collector, and the resistance of the electrolyte solution. ${ }^{51}$ Electrochemical impedance spectroscopy of the HPC-DK-1.0 presents a short Warburg. region and a small semicircle diameter, which means that its hierarchical porous structure favors electrolyte ion access and rapid ion transportation. The $R_{\mathrm{S}}$ value of HPC-DK-1.0 is the smallest among the three HPC carbon electrodes, which indicates its excellent conductivity and improves its supercapacitive performance.

The symmetric supercapacitor was assembled by using HPCDK-1.0 as positive and negative electrode materials, because the three-electrode configuration may produce large errors, and lead to an overestimation of capacitance. Fig. 7a shows typical CV curves of the HPC-DK-1.0 based electrode over scanning rates of 5-200 $\mathrm{mV} \mathrm{s}^{-1}$ in a $6.0 \mathrm{M} \mathrm{KOH}$ aqueous electrolyte. All CV curves were rectangular without obvious redox peaks at a scanning rate of $5-200 \mathrm{mV} \mathrm{s}^{-1}$, which is characteristic of excellent capacitive behavior. ${ }^{52}$ No significant distortions in the CV curves result when the scan rate was increased to $200 \mathrm{mV} \mathrm{s}^{-1}$, which suggests rapid ion/charge transport within electrodes and the near-ideal capacitive behavior with a good rate capability. 

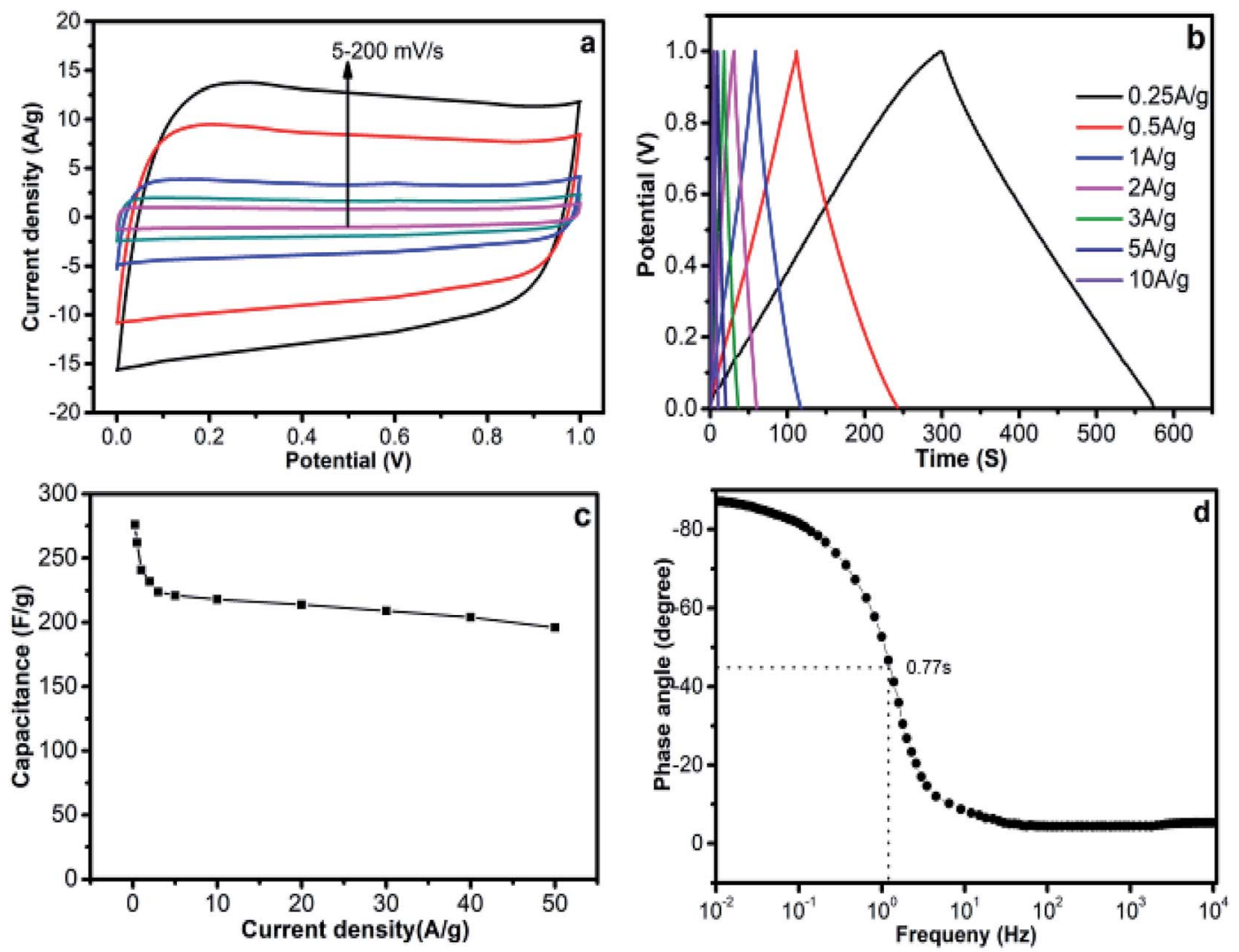

Fig. 7 Capacitive performance of symmetric electrode for HPC-DK-1.0 in 6.0 M KOH aqueous electrolyte. (a) CV curves at different scanning rates, (b) charge-discharge profiles at different current densities, (c) capacitance retention at different current densities, and (d) Bode plot of phase angle verses frequency.

Fig. 7b shows the galvanostatic charge-discharge curves of HPC-DK-1.0 based supercapacitor at different current densities from 0.25 to $10 \mathrm{~A} \mathrm{~g}^{-1}$. The symmetric linear charge and discharge curves with a negligible voltage drop demonstrate a high coulombic efficiency and a negligible internal resistance. From the discharge curve, the specific capacitance at a constant current density of $0.25 \mathrm{~A} \mathrm{~g}^{-1}$ was found to be $276 \mathrm{~F} \mathrm{~g}^{-1}$, which is much higher than that of the RGO-CMK-5 electrode $\left(144.4 \mathrm{~F} \mathrm{~g}^{-1}\right.$ at $\left.0.2 \mathrm{~A} \mathrm{~g}^{-1}\right),{ }^{53}$ the curved graphene electrode $\left(154.1 \mathrm{~F} \mathrm{~g}^{-1}\right.$ at

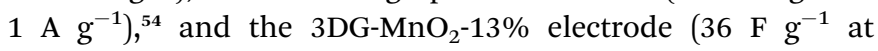
$\left.0.5 \mathrm{~A} \mathrm{~g}^{-1}\right)^{55}$ in a two-electrode system. The capacitance could retain a high value of $196 \mathrm{~F} \mathrm{~g}^{-1}$ even at a very high current density of $50 \mathrm{~A} \mathrm{~g}^{-1}$ (Fig. 7c), which indicates the high rate performance of the HPC-DK-1.0 based symmetric supercapacitor. The specific capacitance of HPC-DK-1.0 at different current densities is shown in Fig. 7c. The capacitance decreases rapidly from 256 to $224 \mathrm{~F} \mathrm{~g}^{-1}$ when the current density increases from 0.25 to $3 \mathrm{~A} \mathrm{~g}^{-1}$. After that, it drops slowly at high current densities from 3 to $50 \mathrm{~A} \mathrm{~g}^{-1}$, which reveals the excellent rate capability of the HPC-DK-1.0 electrodes. Such an excellent rate performance is essential for practical application involving a high-rate supercapacitor. The kinetic ion diffusion within the electrode was investigated by electrochemical impedance spectroscopy. Fig. 7d shows the dependence of the impedance phase angle on the frequency of the HPC-DK-1.0 electrode. The relaxation time constant $\tau_{0}$ of the supercapacitor, which is defined as $1 / f_{0}$ at a phase angle of $-45^{\circ}$, represents the point where the resistive and capacitive impedances are equal. For the symmetric supercapacitor that was fabricated from HPC-DK1.0, the characteristic frequency $f_{0}$ at a phase angle of $-45^{\circ}$ was observed to be $1.3 \mathrm{~Hz}$ in $\mathrm{KOH}$ aqueous electrolyte, which corresponds to a time constants $\tau_{0}$ of $0.77 \mathrm{~s}$, which is nearly equal to $0.73 \mathrm{~s}$ of the graphene aerogel of the GA-0.5 electrode, ${ }^{56}$ and is smaller than that of a conventional activated carbonbased electrode $(10 \mathrm{~s}) .^{57}$ The very short time constant of HPCDK-1.0 highlights the critical role of nanopores in promoting the ion kinetic diffusion in the interior of the electrodes.

Cycling stability is one of the most important parameters for practical application of supercapacitors. The cycling stability of an HPC-DK-1.0 based supercapacitor was investigated by a consecutive charge-discharge measurement at a constant current density of $3 \mathrm{~A} \mathrm{~g}^{-1}$ for 10000 cycles. Although the specific capacitance of the HPC-DK-1.0 electrode decreased gradually with the cycling number (Fig. 8a), a capacitance retention of $92.9 \%$ was still obtained after 10000 cycles, which indicates its good electrochemical stability. The rectangular CV profile (Fig. 8a inset) and Nyquist plots (Fig. 8b) with negligible changes after 10000 cycles support this electrochemical cyclability. The small semicircle in the high-frequency region and the almost vertical line in the low-frequency region indicate that 

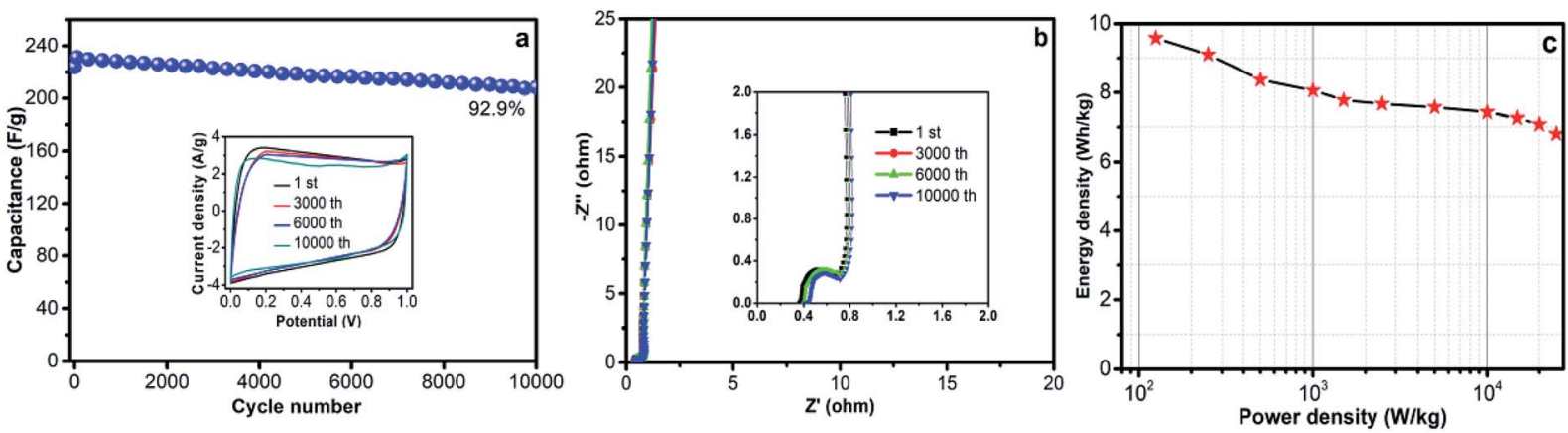

Fig. 8 (a) CV curves at different cycles, (b) Nyquist plots of different cycles with the inset showing the plots in the high frequency region, and (c) Ragone plot.

HPC-DK-1.0 has an excellent electrical conductivity (Fig. 8b inset). The capability of HPC-DK-1.0 that integrated its high rate performance with an excellent cycling stability is of great importance for high-performance supercapacitors. Fig. 8c shows the Ragone plot of the symmetric capacitor. With $\mathrm{KOH}$ as the electrolyte, the energy and the power density were $6.81 \mathrm{~W} \mathrm{~h} \mathrm{~kg}^{-1}$ and $25 \mathrm{~kW} \mathrm{~kg}^{-1}$ at a current density of $50 \mathrm{~A} \mathrm{~g}^{-1}$, respectively, which exhibits an outstanding power performance.

\section{Conclusions}

We have developed a simple and effective template-free method to prepare HPCs by constructing diketone cross-linking bridges in RFCP. The oxygen facilitated extensive cross-linking formation and prevented graphitization. The diketone structure in the pitch is unstable and can decompose to $\mathrm{CO}_{2}$ and $\mathrm{CO}$ during heat treatment, which can assist in creating additional pores. The $S_{\mathrm{BET}}$ and $V_{\text {tot }}$ of the HPCs increased with the cross-linking agent to precursor ratio. The optimum cross-linking agent to precursor ratio was found to be 1.0, which resulted in a specific surface area of $1394.6 \mathrm{~m}^{2} \mathrm{~g}^{-1}$ and a porosity volume of $1.54 \mathrm{~cm}^{3}$ $\mathrm{g}^{-1}$. HPC-DK-1.0 could be one of the best electrode materials for supercapacitors with a high specific capacitance of $276 \mathrm{~F} \mathrm{~g}^{-1}$ at a current density of $0.25 \mathrm{~A} \mathrm{~g}^{-1}$ and a high capacitance retention of $92.9 \%$ after 10000 cycles in a symmetric two-electrode cell because of its high surface area, small inner resistance and high electrical conductivity. These results show that these porous carbon materials are promising for use in high-performance supercapacitors.

\section{Conflicts of interest}

There are no conflicts to declare.

\section{References}

1 H. J. Zeng, W. Wang, J. Li, J. Luo and S. L. Chen, ACS Appl. Mater. Interfaces, 2018, 10, 8721-8729.

2 Q.-M. Ji, S.-B. Yoon, J. Hill, A. Vinu, J.-S. Yu and K. Ariga, J. Am. Chem. Soc., 2009, 131, 4220-4221.
3 Z. C. Lou, Y. J. Li, H. Han, H. H. Ma, L. Wang, J. B. Cai, L. T. Yang, C. L. Yuan and J. Zou, ACS Sustainable Chem. Eng., 2018, 11, 15598-15607.

4 B.-Z. Fang, J.-H. Kim, M.-S. Kim, A. Bonakdarpour, A. Lam, D. Wilkinson and J.-S. Yu, J. Mater. Chem., 2012, 22, 1903119038.

5 B.-Z. Fang, J.-H. Kim, M.-S. Kim and J.-S. Yu, Chem. Mater., 2009, 21, 789-796.

6 W. Geng, F. Ma, G. Wu, S. Song, J. Wan and D. Ma, Electrochim. Acta, 2016, 191, 854-863.

7 H. Feng, M. Zheng, H. Dong, Y. Xiao, H. Hu, Z. Sun, C. Long, Y. Cai, X. Zhao, H. Zhang, B. Lei and Y. Liu, J. Mater. Chem. A, 2015, 29, 15225-15234.

8 O. Martin, B. Lars, P. Katja, T. Sören, R. Martin, L. M. H. Rümmeli, E. Jürgen, A. Eychmüller and K. Stefan, Adv. Energy Mater., 2014, 4, 130645-130654.

9 X. M. Ma, M. X. Liu, L. H. Gan, Y. H. Zhao and L. W. Chen, J. Solid State Electrochem., 2013, 17, 2293-2301.

10 Y. Guo, Z.-Q. Shi, M.-M. Chen and C.-Y. Wang, J. Power Sources, 2014, 252, 235-243.

11 S. Song, F. Ma, G. Wu, D. Ma, W. Geng and J. Wan, J. Mater. Chem. A, 2015, 3, 18154-18162.

12 J. Deng, T. Xiong, F. Xu, M. Li, C. Han, Y. Gong, H. Wang and Y. Wang, Green Chem., 2015, 17, 4053-4060.

13 X. Yang, W. Zhu, G. Cao and X. Zhao, RSC Adv., 2016, 6, 7159-7171.

14 J. Yang, K. Nakabayashi, J. Miyawaki and S. H. Yoon, Carbon, 2016, 106, 28-36.

15 Y. Yang, Q. Lin, Y. Huan and D. Guo, J. Anal. Appl. Pyrolysis, 2011, 91, 310-315.

16 B. Tsyntsarski, B. Petrova, T. Budinova, N. Petrov, M. Krzesinska, S. Pusz, et al., Carbon, 2010, 48, 3523-3530.

17 W. Q. Li, A. J. Zhang, H. Gao, M. J. Chen, A. H. Liu, H. Bai and L. Li, Chem. Commun., 2016, 52, 2780-2783.

18 C. Long, X. Chen, L. Jiang, L. Zhi and Z. Fan, Nano Energy, 2015, 12, 141-151.

19 W. Geng, F. Ma, G. Wu, S. Song, J. Wan and D. Ma, Electrochim. Acta, 2016, 191, 854-863.

20 X. Zheng, J. Luo, W. Lv, D.-W. Wang and Q.-H. Yang, Adv. Mater., 2015, 27, 5388-5395.

21 X. He, H. Zhang, H. Zhang, X. Li, N. Xiao and J. Qiu, J. Mater. Chem. A, 2014, 2, 19633-19640. 
22 Q. Wang, J. Yan, T. Wei, J. Feng, Y. Ren, Z. Fan, M. Zhang and X. Jing, Carbon, 2013, 60, 481-487.

23 W. Li, A. Zhang, H. Gao, M. Chen, A. Liu, H. Bai and L. Li, Chem. Commun., 2016, 13, 2780-2783.

24 H. Gao, L. Ding, H. Bai, A. Liu, S. Li and L. Li, J. Mater. Chem. A, 2016, 42, 16490-16498.

25 G. Rosebrock, A. Elgafy, T. Beechem and K. Lafdi, Carbon, 2005, 43, 3075-3087.

26 Y. Luo, S. Zhang, Y. Ma, W. Wang and B. Tan, Polym. Chem., 2013, 4, 1126-1131.

27 B. K. Pradhan and N. K. Sandle, Carbon, 1999, 37, 1323-1332.

28 P. Kleszyka, P. Ratajczaka, P. Skowrona, J. Jagiellob, Q. Abbasa, E. Frackowiaka and F. éguin, Carbon, 2015, 8, 148-157.

29 C. Bommiera, R. X. W. Wanga, X. F. Wanga, D. Wena, J. Lub and X. L. Ji, Nano Energy, 2015, 13, 709-717.

30 T. I. T. Okpalugo, P. Papakonstantinou, H. Murphy, J. McLaughlin and N. M. D. Brown, Carbon, 2005, 43, 153161.

31 J.-H. Zhou, Z.-J. Sui, J. Zhu, P. Li, D. Chen, Y.-C. Dai and W.-K. Yuan, Carbon, 2007, 45, 785-796.

32 J. A. Monge, D. C. Amoros, A. Gungor and L. Toppare, Fuel, 2001, 80, 41-48.

33 H. M. Zhu, J. H. Yan, X. G. Jiang, Y. E. Lai and K. F. Cen, J. Hazard. Mater., 2008, 153, 670-676.

34 M. Acik, C. Mattevi, C. Gong, G. Lee, K. Cho, M. Chhowalla and Y. J. Chabai, ACS Nano, 2010, 4, 5861.

35 M. Pérez, M. Granda, R. Santamaría, et al., Fuel, 2004, 83, 1257-1265.

36 Q. Lin, T. Li, Y. Ji, et al., Fuel, 2005, 84, 177-182.

37 Y. Martin, R. Garcia, R. A. Sole and S. R. Moinelo, Energy Fuels, 1996, 10, 436-442.

38 Y. Martin, R. Garcia, P. Keating, C. E. Snape and S. R. Moinelo, Energy Fuels, 2000, 14, 380-392.

39 M. Dumont, G. Chollon, M. A. Dourges, R. Pailler, X. Bourrat, R. Naslain, J. L. Bruneel and M. Couzi, Carbon, 2002, 40, 1475-1486.
40 M. J. Prauchner, V. M. D. Vanya, N. D. S. Molhallem, C. Otani, S. Otani and L. C. Pardini, Biomass Bioenergy, 2005, 28, 53-61.

41 W. Feng, P. He, S. Ding, G. Zhang, M. He, F. Dong, et al., RSC Adv., 2016, 6, 5949-5956.

42 A. Arami-Niya, T. E. Rufford and Z. Zhu, Carbon, 2016, 103, 115-124.

43 C. Chen, E. B. Kennel, A. H. Stiller, P. G. Stansberry and J. W. Zondlo, Carbon, 2006, 44, 1535-1543.

44 L.-L. Zhang, H.-H. Li, Y.-H. Shi, C.-Y. Fan, X.-L. Wu, H.-F. Wang, H.-Z. Sun and J.-P. Zhang, ACS Appl. Mater. Interfaces, 2016, 8, 4233-4241.

45 Y. Han, X. T. Dong, C. Zhang and S. X. Liu, J. Power Sources, 2013, 227, 118-122.

46 M.-S. Park, S. Cho, E. Jeong and Y.-S. Lee, J. Ind. Eng. Chem., 2015, 23, 27-32.

47 T. Guan, K. Li, J. Zhao, R. Zhao, G. Zhang, D. Zhang and J. Wang, J. Mater. Chem. A, 2017, 5, 15869-15878.

48 J. Gong, B. Michalkiewicz, X. Chen, E. Mijowska, J. Liu, Z. Jiang, X. Wen and T. Tang, ACS Sustainable Chem. Eng., 2014, 2, 2837-2844.

49 L. Wang, J. Wang, F. Jia, C. Wang and M. Chen, J. Mater. Chem. A, 2013, 1, 9498-9507.

50 T. E. Rufford, D. Hulicova-Jurcakova, K. Khosla, Z. Zhu and G. Q. Lu, J. Power Sources, 2010, 195, 912-918.

51 L. Lai, H. Yang, L. Wang, B. K. Teh, J. Zhong, H. Chou, L. Chen, W. Chen, Z. Shen, R. S. Ruoff and J. Lin, ACS Nano, 2012, 6, 5941-5951.

52 M. Inagaki, H. Konno and O. Tanaike, J. Power Sources, 2010, 195, 7880-7903.

53 Z. Lei, Z. Liu, H. Wang, X. Sun, L. Lu and X. Zhao, J. Mater. Chem. A, 2013, 1, 2313-2321.

54 C. Liu, Z. Yu, D. Neff, A. Zhamu and B. Z. Jang, Nano Lett., 2010, 10, 4863-4868.

55 X. Sun, H. Wang, Z. Lei, Z. Liu and L. Wei, RSC Adv., 2014, 4, 30233-30240.

56 X. Sun, P. Cheng, H. Wang, H. Xu, L. Dang, Z. Liu and Z. Lei, Carbon, 2015, 92, 1-10.

57 Y. Gogotsi and P. Simon, Science, 2011, 334, 917-918. 